\title{
Epithelioid Angiosarcoma of Bone: A Neoplasm with Potential Pitfalls in Diagnosis
}

\author{
Jie Xu\#, Ruo-Fan Ma ${ }^{\# *}$, Deng Li, Liang-Ping Li, Zhi-Qing Cai, Wen-Wu Dong, Yan Chen, Yue Ding
}

Department of Orthopaedics, Sun Yat-sen Memorial Hospital, Sun Yat-sen University, Guangzhou, China.

Email: *maruofan_sysu@yahoo.com.cn

Received July $16^{\text {th }}, 2012$; revised August $18^{\text {th }}, 2012$; accepted August $26^{\text {th }}, 2012$

\begin{abstract}
Angiosarcoma of bone is an exceedingly rare primary bone malignancy that can present as an aggressive osteolytic lesion. This subset can radiologically mimic non-vascular neoplasms and impose serious challenges in reaching the correct diagnosis. Meanwhile histological diagnosis can be extremely challenging too, as the pathological features often resemble that of aneurysmal bone cysts. We present an unusual case of a 22 -year-old woman who presented with a rapidly growing humeral tumor of 8 months' duration. The case of intraosseous angiosarcoma presented as a diagnostic dilemma and the relevant radiological and pathologic findings were discussed. We describe the clinical, radiological and pathological features of this unique case, and review the literature concerning Angiosarcoma of bone. Our case highlights the diagnostic difficulties for such very rare tumours and clinico-pathological correlation is of paramount importance to differential diagnosis.
\end{abstract}

Keywords: Angiosarcoma; Bone Neoplasms; Vascular Tissue Neoplasms; Aneurysmal Bone Cysts; Pitfall

\section{Introduction}

Angiosarcoma is an uncommon neoplasm characterized by rapidly proliferating, extensively infiltrating anaplastic cells derived from blood vessels and lining irregular blood-filled spaces. Angiosarcomas are aggressive and tend to recur locally and spread widely [1]. Approximately $6 \%$ of all angiosarcomas are found in bone [2]. Primary angiosarcoma arising in bone is very rare, and accounts for less than $1 \%$ of malignant bone tumours [3]. Skeletal angiosarcoma generally affects young adults and elderly individuals, with a $2 / 1$ male to female ratio [4]. The bones of the lower limb, particularly the femur and tibia, are most commonly involved, followed by pelvis, vertebral column and bones of the upper limb [4].

\section{Case Report}

A 22-year-old woman presented with a one-week history of a markedly pain below and lateral to her left shoulder, impeding the patient's sleep and daily life, and being not relieved with common analgesics. She had no history of trauma, and her past medical history was unremarkable. Physical examination revealed a deep tenderness in the region of the proximal humerus; no mass was tender to

\footnotetext{
*Corresponding author.

${ }^{\#}$ Both authors contributed equally to this work and share the first authorship.
}

palpation. The results of routine laboratory tests were normal. Imaging studies included anteroposterior radiographs (Figure 1(A)), radionuclide Tc-99m methylene diphosphonate bone scan (Figure 1(D)), computed tomo-

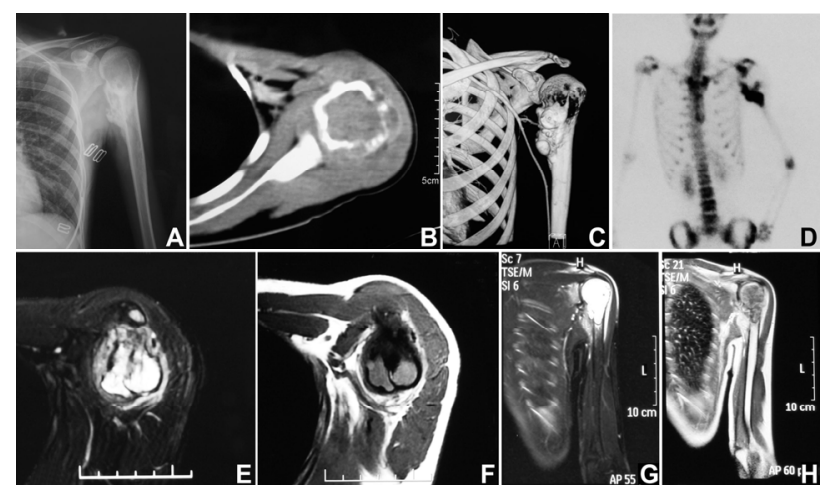

Figure 1. (A) Radiograph of the shoulder showed a welldefined lesion with radiodense margins in the epiphysis of the humerous head, with slight extension into the metaphysis. CT scan revealed an osteolytic lesion with endosteal scalloping and cortical thinning; (B) Axial sequence and (C) 3-D reconstruction; (D) A delayed image from a Tc-99m methylene diphosphonate radionuclide bone scan shows marked uptake of radiotracer. MRI of the left shoulder showing an intermediate to hyperintense lobulated mass with small fluid-filled cavities at the proximal part of the humerus; (E) Axial T1; (F) Axial T2 weighted; (G) Coronal $\mathrm{T} 1$ and $(\mathrm{H})$ Coronal $\mathrm{T} 2$ weighted sequences. 
(CT) scan (Figures 1(B) and (C)), and magnetic resonance imaging (MRI) (Figures 1(E)-(H)). Plain radiographs and computed tomography showed partial destruction of the proximal humerus. Magnetic resonance imaging revealed a lesion with high intensity on T1- weighted images and intermediate intensity on T2-weighted images. The differential diagnosis for the lesion on the upper limb at the time of visit included aneurismal bone cyst, fibrousdysplasia, osteosarcoma and angiosarcoma. The lesion was biopsied to confirm the diagnosis. A fine needle aspiration biopsy of the lesion was taken. A diagnosis was not obtained at that time as a result of an inadequate tissue sample for proper histologie identification. Intralesional curettage with burring was performed and lavage with hydrogen peroxide. Bone graft was packed into the cavity of the lesion to reinforce the intertuberositic region (Figure 2(A)). Histological analysis of the resected specimen showed cystic spaces which were lined by fibrous septa that contained blood vessels. Prominent, multinucleated giant cells were seen and there were areas of haemosiderin deposition and recent haemorrhage. No other accompanying neoplastic cells were seen. Therefore, given these features, a provisional diagnosis of cystic haemorrhagic aneurysmal bone cyst was made (Figure 3(A)). The patient received rehabilitative exercise postoperatively, however, the shoulder pain was persistent. One month after the index operation, she presented with an acute haematoma of the shoulder after a slighter collision. Plain radiograph and CT was performed and revealed pathological fracture in the proximal part of humerus (Figures 2(B) and (C)). Computed tomographic angiography (CTA) of the left arm revealed extravasating collateral branches from the axillary artery around the shoulder (Figure 2(D)). Magnetic resonance imaging shows a contrast-enhancing destructive lesion with soft tissue invasion at the proximal humerus. Welldefined mass with lobular inhomogeneous intermediate to low signal intensity was shown, abutting the pathological fracture (Figures 2(F)-(H)). Around the bloc resection of the proximal humerus was performed. A custom-made titanium prosthesis was used for reconstruction of a mobile joint (Figure 2(E)). A diagnosis of aneurismal bone cyst of bone was made histopathologically (Figures 3(B) and (C)). Three months later, she presented with an enlarging mass in her left shoulder with pain and difficulty in movement (Figure 4(A)). An incisional biopsy was done. Microscopic examination revealed composition of varying size and shaped anastomosing and vasoformative channels lined by stratified spindle-shaped cells with crowded, vesicular, pleomorphic nuclei and sparse nuclei and eosinophilic cytoplasm. The malignant cells formed solid sheets and islands with abortive channels containing red blood cells. Numerous mitoses were present in the malignant cells (Figure 3(D)). Immunohistochemical stains of the malignant cells were strongly positive for CD31 and CD34. The neoplastie cells stained negative for keratin AE1/AE3, CD68 and S-100. A final diagnosis of epithelioid angiosareoma of the humerus was made based on histomorphology and immunohistochemical reactions (Figures 3(E) and (F)). Since angiosarcoma has a high risk of recurrence and metastasis, therefore, after the final pathology reading of angiosarcoma, a radical excision (disarticulation of shoulder girdle) was performed after local radiation (Figures 4(B) and $(\mathbf{C})$ ). She has been followed up and adjuvant chemotherapy and local radiation were performed.

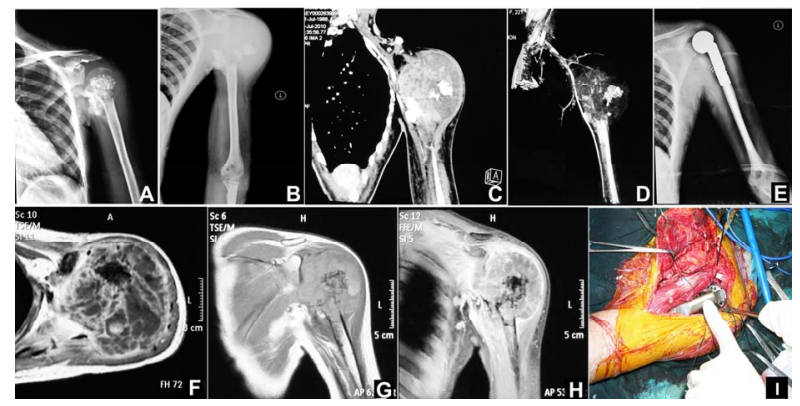

Figure 2. (A) Radiograph of the humerus was performed 1 week after filling of the cavity with bone graft; (B) Radiograph of the humerus 1 month after surgery and a fracture was shown; (C) CT scans reveal the marked destruction of the cortex and a large soft tissue swelling involving the muscles; (D) CTA of the left shoulder was performed and revealed extravasating collateral branches from the axillary artery around the shoulder joint. MRI showed break in the cortex and lesion extending into the muscle; (F) Axial T2 weighted; (G) Coronal T2 and (H) Coronal T1 weighted sequences; (I) Wide resection was performed with custommade titanium prosthesis replacement of proximal left humerus; (E) A postoperative anteroposterior view of X-ray.

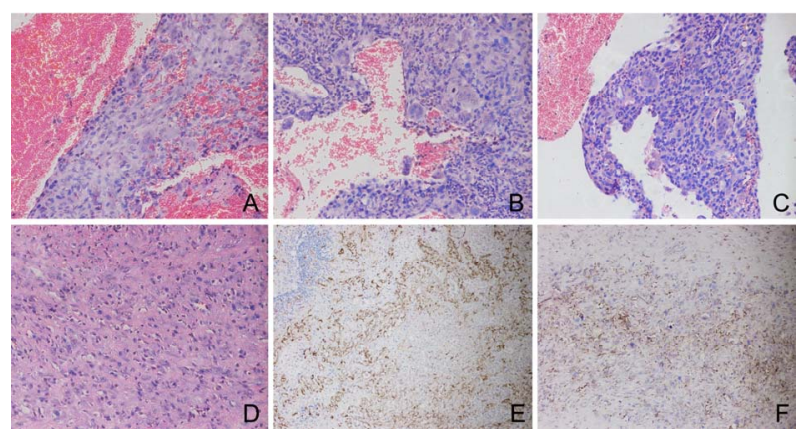

Figure 3. (A)-(C) showing a typical picture of aneurysmal bone cyst. Cavernomatous spaces lined with endothelial cells and containing extravasated red blood cells. Note the multi-nucleated giant cells (Stain, hematoxylin and eosin; original magnification, $\times 200$ ); (D) Solid nests or large sheets of spindle or epithelioid cells are interspersed between and around preexisting vessels with flat endothelium (Stain, hematoxylin and eosin; original magnification, $\times 200)$; (E) Immunohistochemical analysis revealed strong staining of tumor cells for CD31 (Original magnification, $\times 100)$; (F) Immunostaining for CD34 was partially positive (Original magnification, $\times 100)$. 


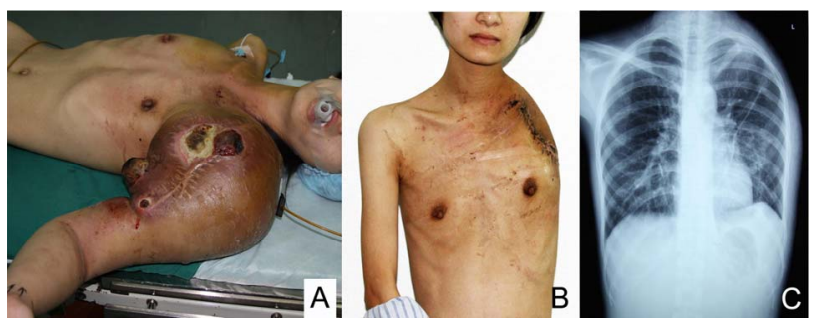

Figure 4. (A) Preoperative view: an enlarging mass presented in the left shoulder; (B) Postoperative view after disarticulation of shoulder girdle; (C) Postoperative anterior- posterior plain radiograph.

\section{Disscussion}

Osseous angiosarcoma is a rare malignant tumor. The etiology of angiosarcoma is still unclear but multifactoral. External beam radiation and bone infarct have been regarded as probable risk factors $[5,6]$. Angiosarcoma of the humerus is extremely rare with only 5 cases reported to date in the English literature since 1953 [4,7-10] (Table 1). To our knowledge, the present case is the sixth case reported. It is more common in men than women, with a ratio of $2: 1$. The majority of patients are affected in the third, fourth and fifth decades of life. However, the tumors are observed in all ages [11-14]. Local pain and swelling are the two most characteristic findings and may last for months or even years [15]. The lesions vary in size and are either medullary or cortical. They may be well defined or poorly defined and may show some degree of expansion. Angiosareoma is a often difficult tumor to diagnose, representing less than 1 percent of all sarcomas [16]. Angiosarcomas are occasionally misdiagnosed initially or considered benign growths. They are only correctly diagnosed months to years later when the tumor has become large, invasive, or developed distant metastasis. The initial closed and open biopsies were not definitive. Due to the unusual nature of this case after the first operation, an open biopsy was performed that also suggested the presence of a benign vascular process. It was because of ongoing symptoms and bony destruction that we elected to resect the proximal humerus, and from representative tissue sections, the diagnosis of aneurysm bone cyst was made. The case is a reminder of the possibility of misinterpreting a benign appearance by imaging. From initial imaging, both the CT scan and MRI, suggest isolated proximal humeral disease with no radiological abnormalities evident elsewhere in the skeletal system. Radiographs imaging findings of angiosarcoma are relatively non-specific. For angiosareoma, radiographs of soft-tissue lesions usually reveal only a non-specific mass. Osseous lesions are predominatly lytic and may reveal a honey-comb or hole-within-hole appearance similar to that of aneurysmal bone cysts (ABCs). Sometimes, a destruction of the bone cortex and periosteal reaction can
Table 1. Angiosarcoma occurring in humerus.

\begin{tabular}{cccc}
\hline Source & Age(yr)/Sex & Treatment & Outcome \\
\hline T. Hasegaw et al. [4] & $48 / \mathrm{M}$ & Wild resection & DOD \\
S. Mittal et al. [5] & $67 / \mathrm{M}$ & Wild resection & DF at 5 yr \\
& & Replacement & \\
& & Chemotherapy & \\
J. Fukuroku et al. [6] & $55 / \mathrm{M}$ & Wild resection & DOD \\
$\begin{array}{c}\text { G. Voggenreiter } \text { et al. } \\
\text { [7] }\end{array}$ & $12 / \mathrm{M}$ & Wild resection & DF at 7 yr \\
K. T. Chen [8] & $87 / \mathrm{M}$ & Wild resection & Absence \\
\hline
\end{tabular}

be seen $[17,18]$. That can also be found in different grades in many other types of lesions: ossifying fibroma [19-21], metastases, myeloma, lymphoma, osteosarcoma, fibrosarcoma or even in fractures [20,22]. CT findings are usually nonspecific [23], consisiting of a soft-tissue mass with attenuation similar to that of muscle, althrough the mass usually markedly enhances with contrast material. MR imaging charateristics, like those of $\mathrm{CT}$, may also be nonspecific. In the experience of Murphey et al. [24], however, prominent serpentine vessels can be identified in some cases, and these findings should suggest the diagnosis. The signal intensity of these vascular structures may reflect either high flow (low signal intensity on images of all pulse sequences) or low flow (high signal intensity on T2-weighted images). Histologically, the features of angiosarcomas of bone can often resemble ABCs [25]. As seen in this case, the histologic features of aneurysmal bone cyst can mimic that of angiosarcoma. On histopathology, ABCs are blood-filled cystic cavities that are lined by a thick, fleshy membrane that has an endothelial-like inner layer. Meanwhile, the majority of ABCs have good responses to primary treatment [26]. However, the clinical course deteriorated progressively which was not compatible to the disease progression of $\mathrm{ABCs}$ in our case. So, the histological diagnosis of ABCs in the initial stage of this case, was secondary one to angiosarcomas. The real course was neglected. Actually, almost $50 \%$ of $\mathrm{ABCs}$ occur as a secondary lesion in another tumor. These lesions include giant cell tumor, chondroblastoma, osteoblastoma and some malignant tumors [27]. Often, the secondary lesions may be actually larger than the associated primary tumor [28]. And that would delay the correct diagnosis, especially for those, which real lesion was not included in the histological section. Thus, secondary ABCs with underlying malignancy should be ruled out especially in adult with rapid progression of disease as demonstrated in our case. It is advisable to use multiple endothelial markers for the immunohistochemical diagnosis of angiosarcoma. CD31 is considered the most sensitive and specific routine marker 
for all types of angiosarcoma [29]. CD34 is another most sensitive and specific marker, whereas CD68 can help to differentiate it from malignant histiocytoma and epithelial membrane antigen can exclude epithelial carcinoma. Therefore, in our case, immunohistochemical staining of vimentin, CD34, CD68, and epithelial membrane antigen were performed. The results showing strong staining of tumor cells for vimentin (Figure 3(C)), partial positivity for CD34 (Figure 3(D)), and negativity for CD68 and epithelial membrane antigen were helpful for the differential diagnosis. No isolated method seems to fully confirm angiosarcoma, of which the diagnosis is really a clinico-radiologic-pathological one. The treatment of angiosarcoma is similar to that of other bone sarcomas, involving radical resection or amputation, the mainstay. Radiation therapy and chemotherapy are now considered helpful [5].

\section{Conclusion}

Angiosarcoma of bone is a rare primary bone sarcoma. The diagnosis is often delayed because of the non-specific clinical presentation and radiological features. Clinico-radiologic-pathological correlation is of paramount importance to differentiate aneurysmal bone cyst from the aggressive type of vascular malignancies.

\section{Consent}

Written informed consent was obtained from the patient for publication of this case report and any accompanying images.

\section{Disclosure Statement}

All authors do not have a conflict of interest.

\section{REFERENCES}

[1] R. Volpe and A. Mazabraud, "Hemangioendothelioma (Angiosarcoma) of Bone: A Distinct Pathologic Entity with an Unpredictable Course?" Cancer, Vol. 49, No. 4, 1982, pp. 727-736.

[2] S. W. Weiss and J. R. Goldblum, "Enzinger and Weiss's Soft Tissue Tumors," Mosby, St. Louis, 2001.

[3] K. K. Unni, "Dahlin's Bone Tumors: General Aspects and Data on 11087 Cases," Lippincott-Raven, Philadelphia, 1996.

[4] T. Hasegawa, Y. Fujii, K. Seki, P. Yang, T. Hirose, K. Matsuzaki and T. Sano, "Epithelioid Angiosarcoma of Bone," Human Pathology, Vol. 28, No. 8, 1997, pp. 985989. doi:10.1016/S0046-8177(97)90016-2

[5] Y. Saglik, Y. Yildiz, H. Atalar and K. Basarir, "Primary Angiosarcoma of the Fibula: A Case Report," Acta Orthopaedica Belgica, Vol. 73, No. 6, 2007, pp. 799-803.

[6] L. A. Cerilli and R. E. Fechner, "Angiosarcoma Arising in a Bone Infarct," Annals of Diagnostic Pathology, Vol.
3, No. 6, 2007, pp. 370-373. doi: 10.1016/S1092-9134(99)80016-1

[7] S. Mittal, C. Goswami, N. Kanoria and A. Bhattacharya, "Post-Irradiation Angiosarcoma of Bone," Journal of Cancer Research and Therapeutics, Vol. 3, No. 2, 2007, pp. 96-99. doi:10.4103/0973-1482.34687

[8] J. Fukuroku, K. Kusuzaki, H. Murata, S. Nakamura, H. Takeshita, M. Hirata, S. Hashiguchi and Y. Hirasawa, "Two Cases of Secondary Angiosarcoma Arising from Fibrous Dysplasia," Anticancer Research, Vol. 19, No. 5C, 1999, pp. 4451-4457.

[9] G. Voggenreiter, S. Assenmacher and K. P. Schmit-Neuerburg, "Tikhoff-Linberg Procedure for Bone and Soft Tissue Tumors of the Shoulder Girdle," Archives of Surgery, Vol. 134, No. 3, 1999, pp. 252-257. doi:10.1001/archsurg.134.3.252

[10] K. T. Chen, "Hemangiosarcoma Complicating Paget's Disease of the Bone," Journal of Surgical Oncology, Vol. 28, No. 3, 1985, pp. 187-189. doi:10.1002/jso.2930280308

[11] H. D. Dorfman, G. C. Steiner and H. L. Jaffe, "Vascular Tumors of Bone," Human Pathology, Vol. 2, No. 3, 1971, pp. 349-376. doi:10.1016/S0046-8177(71)80004-7

[12] K. K. Unni, J. C. Ivins, J. W. Beabout and D. C. Dahlin, "Hemangioma, Hemangiopericytoma, and Hemangioendothelioma (Angiosarcoma) of Bone," Cancer, Vol. 27, No. 6, 1971, pp. 1403-1414.

[13] C. A. Garcia-Moral, "Malignant Hemangioendothelioma of Bone. Review of World Literature and Report of Two Cases," Clinical Orthopaedics and Related Research, Vol. 82, 1972, pp. 70-79. doi:10.1097/00003086-197201000-00009

[14] L. E. Bohn, L. P. Dehner and H. J. Walker, "Case Report 204. Multicentric Angiosarcoma of Bone Involving the Right Lower Extremity," Skeletal Radiology, Vol. 8, No. 34, 1982, pp. 303-305. doi:10.1007/BF02219628

[15] M. Campanacci, S. Boriani and A. Giunti, "Hemangioendothelioma of Bone: A Study of 29 Cases," Cancer, Vol. 46, No. 4, 1980, pp. 804-814.

[16] F. G. Fedok, R. J. Levin, M. E. Maloney and K. Tipirneni, "Angiosarcoma: Current Review," American Journal of Otolaryngology, Vol. 20, No. 4, 1999, pp. 223-231. doi:10.1016/S0196-0709(99)90004-2

[17] M. M. Kalantar, "Aneurysmal Bone Cysts of the Jaws: Clinicopathological Features, Radiographic Evaluation and Treatment Analysis of 17 Cases," Journal of CranioMaxillofacial Surgery, Vol. 26, No. 1, 1998, pp. 56-62. doi:10.1016/S1010-5182(98)80036-X

[18] M. H. Motamedi and E. Yazdi, “Aneurysmal Bone Cyst of the Jaws: Analysis of 11 Cases," Journal of Oral and Maxillofacial Surgery, Vol. 52, No. 5, 1994, pp. 471-475. doi:10.1016/0278-2391(94)90343-3

[19] S. E. Smith and M. J. Kransdorf, "Primary Musculoskeletal Tumors of Fibrous Origin," Seminars in Musculoskeletal Radiology, Vol. 4, No. 1, 2000, pp. 73-88. doi:10.1055/s-2000-6856

[20] G. Santeusanio, A. Bombonati, U. Tarantino, P. Craboledda, B. Marino, R. Birbe, A. Ortenzi and S. Villaschi, "Multifocal Epithelioid Angiosarcoma of Bone: A Poten- 
tial Pitfall in the Differential Diagnosis with Metastatic Carcinoma," Applied Immunohistochemistry \& Molecular Morphology, Vol. 11, No. 4, 2003, pp. 359-363. doi:10.1097/00129039-200312000-00014

[21] D. E. Wenger and L. E. Wold, "Malignant Vascular Lesions of Bone: Radiologic and Pathologic Features," Skeletal Radiol, Vol. 29, No. 1, 2000, pp. 619-631. doi:10.1007/s002560000261

[22] E. Verhaven, H. De Boeck and P. Opdecam, "Osteosarcoma Appearing as a Pathologic Fracture," Acta Orthopaedica Belgica, Vol. 57, No. 4, 1991, pp. 437-441.

[23] D. M. Coldwell, R. L. Baron and C. Charnsangavej, "Angiosarcoma. Diagnosis and Clinical Course," Acta Radiologica, Vol. 30, No. 6, 1989, pp. 627-631. doi:10.3109/02841858909174727

[24] M. D. Murphey, K. J. Fairbairn, L. M. Parman, K. G. Baxter, M. B. Parsa and W. S. Smith, "From the Archives of the AFIP. Musculoskeletal Angiomatous Lesions: Radiologic-Pathologic Correlation," Radiographics, Vol. 15, No. 4, 1995, pp. 893-917.
[25] H. D. Dorfman and B. Czerniak, "Bone Cancers," Cancer, Vol. 75, No. 1, 1995, pp. 203-210.

[26] H. J. Mankin, F. J. Hornicek, E. Ortiz-Cruz, J. Villafuerte and M. C. Gebhardt, "Aneurysmal Bone Cyst: A Review of 150 Patients," Journal of Clinical Oncology, Vol. 23, No. 27, 2005, pp. 6756-6762. doi:10.1200/JCO.2005.15.255

[27] M. Campanacci, F. Bertoni and P. Bacchini, "Aneurysmal Bone Cyst. In Bone and Soft Tissue Tumors," SpringerVerlag, Vienna, 1990.

[28] L. F. Tse, E. T. Ek, J. L. Slavin, S. M. Schlicht and P. F. Choong, "Intraosseous Angiosarcoma with Secondary Aneurysmal Bone Cysts Presenting as an Elusive Diagnostic Challenge," International Seminars in Surgical Oncology, Vol. 5, 2008, p. 10. doi:10.1186/1477-7800-5-10

[29] B. R. De Young, H. J. Frierson, M. N. Ly, D. Smith and P. E. Swanson, "CD31 Immunoreactivity in Carcinomas and Mesotheliomas," American Journal of Clinical Pathology, Vol. 110, No. 3, 1998, pp. 374-377. 